\title{
Analysis of ancient painting by shortwave infrared imaging spectroscopy
}

\author{
Taixia $\mathrm{Wu}^{1}$, Guanghua $\mathrm{Li}^{2}$, Zehua Yang ${ }^{2}$, Yong $\mathrm{Lei}^{2}$. \\ 1. School of Earth Sciences and Engineering, Hohai University, Nanjing, China \\ 2. The Palace Museum, Beijing, China
}

Currently, non-destructive testing for ancient paintings depends mainly on spectral analysis technology.[1, 2] Multi-spectral and hyperspectral analysis technology can provide analysts with a deeper, clearer, and more intuitive understanding of the color, damage characteristics, pigment composition, and techniques used to make the painting. [3, 4] In this study, an ancient Chinese painting (currently stored in the Forbidden City and numbered Gu-6541) was used as an example. It's imaging spectrum data were obtained at a wavelength of Shortwave infrared (SWIR, 1000-2500 nm) using a ground-based shortwave infrared imaging spectrometer.

Figure 1 shows the shortwave infrared imaging data at 1604nm band and SWIR spectrum curves of clothes and carbon black pigment. The spectral curve of pigment used for clothes and line sketches are totally different at the SWIR band. Usually line sketches are drawn with pigments such as carbon black, which shows strong absorption along all wavelengths in shortwave infrared, contrasted with the pigments with relatively high reflection that resulted with light color in images produced with tested wavelengths, providing contrast marked enough to extract lines drawn with carbon black.

Pigment is the main component of the painting. Here, the azurite was took for example. Azurite, also known as blue copper ore, chemical formula $2 \mathrm{CuCO}_{3} \cdot \mathrm{Cu}(\mathrm{OH})_{2}$. The absorption valleys at SWIR were detected at $2282 \mathrm{~nm}$, and $2351 \mathrm{~nm}$. The main cause of the formation of the three spectral absorption features is the strong absorption of hydroxyl and carbonate in corresponding position, among which the latter two are more characteristic and more helpful in identifying azurite. Batch extraction was performed for pigments used in the ancient painting using SAM (Spectral Angle Mapper) method and supervised classification.

The hidden information in paintings generally includes signs of repair, hidden text patterns, and illegible information. Figre 3 shows the extraction of repaired correction marks. The second principle component (PCA2) emphasized the traces of correction shown in Figure 3 (b). The third principle component (PCA3) showed a large, semi-circular pattern over the hat worn by the figure, which may be used to highlight the correction trace shown in Figure 3(c).

The outcomes can be summarized as: (1) Imaging spectrum data collected using ground-based shortwave infrared imaging spectrometer greatly facilitate the extraction of features of line drafting. (2) SWIR imaging spectrum data can be used to identify and classify mineral pigments. (3) SWIR imaging spectrum data can detect the trace of correction.

\section{References:}

[1] J.-H. Cheng and D.-W. Sun, "Rapid and non-invasive detection of fish microbial spoilage by visible and near infrared hyperspectral imaging and multivariate analysis," LWT-Food Science and Technology, vol. 62 (2015), pp. 1060-1068. 
[2] C. Daffara, E. Pampaloni, L. Pezzati, M. Barucci, and R. Fontana, "Scanning multispectral IR reflectography SMIRR: an advanced tool for art diagnostics," Accounts of chemical research, vol. 43 (2010), pp. 847-856.

[3] J. K. Delaney, E. Walmsley, B. H. Berrie, and C. F. Fletcher, "Multispectral imaging of paintings in the infrared to detect and map blue pigments," Proceedings of the National Academy of sciences, (2005) pp. 120-136.

[4] F. Rosi, C. Miliani, R. Braun, R. Harig, D. Sali, B. G. Brunetti, et al., "Noninvasive analysis of paintings by mid-infrared hyperspectral imaging," Angewandte Chemie, vol. 125 (2013), pp. 5366-5369.
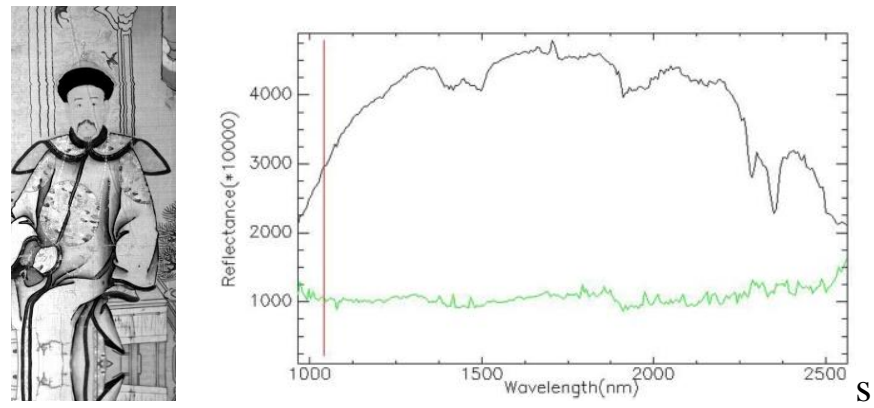

Figure 1. Shortwave infrared imaging data at $1604 \mathrm{~nm}$ and SWIR spectrum curves of clothes and carbon black pigment.

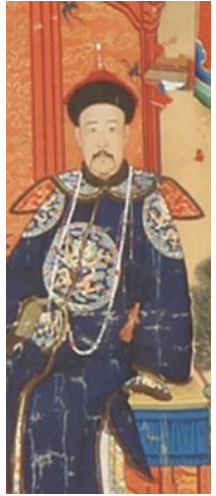

(a)

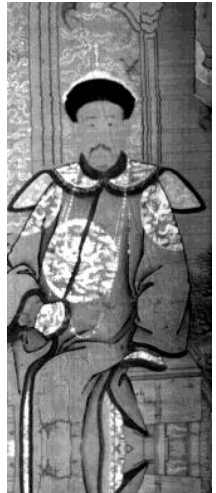

(b)

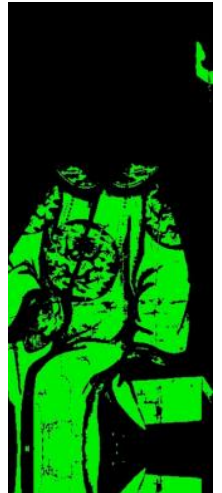

(c)

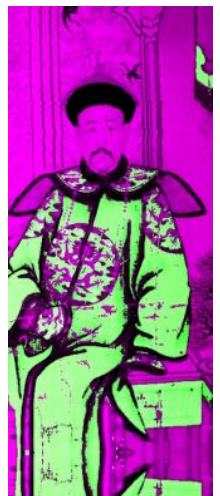

(d)

Figure 2. Extraction of spectral angle mapper for pigment used for clothes (azurite) and background. (a) RGB image. (b) Images produced with wavelengths of $2282 \mathrm{~nm}$. (c) Azurite extraction of clothes with SAM technique. (d) False color composition image with result (c) and $1159 \mathrm{~nm}$ image.

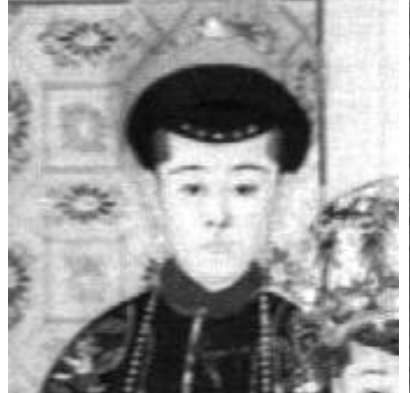

(a)

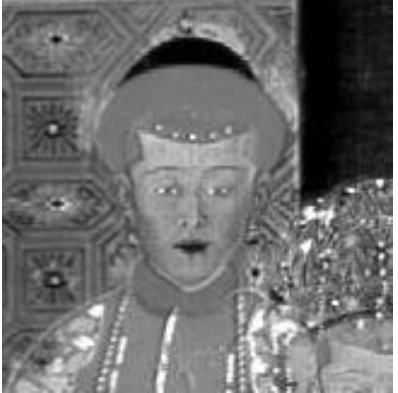

(b)

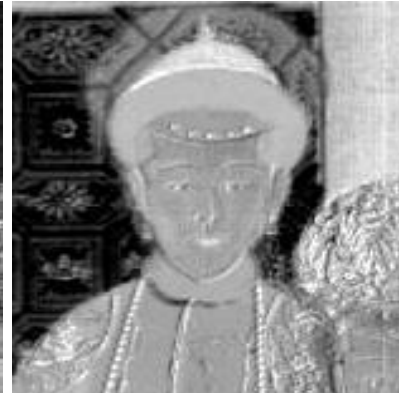

(c)

Figure 3. Extraction of traces of corrections. (a) $1302 \mathrm{~nm}$ image, (b) PCA2 image, (c) PCA3 image. (b) and (c) are images of second and third principle components, which show different signs indicating corrections. 